\title{
Towards Understanding the Origin of Cosmic Ray Electrons with Precision Results from AMS on ISS
}

\author{
Weiwe $\mathbf{X u}^{*}$ \\ Shandong University \\ for the AMS-02 Collaboration ${ }^{\dagger}$ \\ E-mail: Weiwei.Xu@CERN.CH
}

The precision measurements of the cosmic ray electron flux in the energy range from $0.5 \mathrm{GeV}$ to $1.4 \mathrm{TeV}$ based on 28.1 million electrons collected by the Alpha Magnetic Spectrometer on the International Space Station in its first 6.5 years operation. The AMS electron flux exhibits a significant excess starting from $42.1_{-5.2}^{+5.4} \mathrm{GeV}$ compared to the lower energy power law trend. At $5 \sigma$ significance level, the electron flux does not have an exponential cutoff below $1.9 \mathrm{TeV}$. In the entire energy range, the electron flux can be described by the sum of two power law components. The distinctive properties in the electron flux provide new understanding of the origins of cosmic ray electrons.

36th International Cosmic Ray Conference -ICRC2019-

July 24th - August 1st, 2019

Madison, WI, U.S.A.

\footnotetext{
${ }^{*}$ Speaker.

${ }^{\dagger}$ for collaboration list see PoS(ICRC2019)1177
} 


\section{Introduction}

The precision measurement of the electron flux in the primary cosmic rays from $0.5 \mathrm{GeV}$ to 1.4 TeV with the Alpha Magnetic Spectrometer (AMS) on the International Space Station (ISS) is presented. The measurements are based on 28.1 million electron events collected by AMS from May 19, 2011 to November 12, 2017. The results on the behavior of electron flux at high energies are crucial for understanding the origins of high energy cosmic-ray electrons and positrons.

\section{AMS detector and analysis}

The description of the AMS detector is presented in Ref. [1] and references therein. The detector performance on orbit is continuously monitored and the overall AMS instrument response stability is $(0.0 \pm 0.4) \%$. For the details of AMS detectors and advances in the analysis methods please refer to upcoming AMS review article.

AMS has collected $1.07 \times 10^{11}$ cosmic rays in the first 6.5 years of operation. The collection time for galactic cosmic rays increases with rigidity reaching $1.51 \times 10^{8} \mathrm{~s}$ above $30 \mathrm{GeV}$. Template fit methods are used to determine the number of electrons in each energy bin. In total, 28.1 million electrons are collected from $0.5 \mathrm{GeV}$ and $1.4 \mathrm{TeV}$. For the analysis details and particularly the systematic errors please refer to AMS recent publications in PRL [2, 3].

\section{Results}

The AMS results on the electron spectrum (i.e. the flux scaled with $\tilde{E}^{3}, \tilde{E}^{3} \Phi_{e^{-}}$) is presented in Fig. 1 in comparison with the most recent AMS positron spectrum [2]. The spectrally weighted mean energy $\tilde{E}$ is calculated following Ref. [4] for a flux $\propto E^{-3}$. As seen, the electron and positron spectra have distinctly different magnitudes and energy dependencies.

The AMS results on the electron spectrum together with earlier measurements $[5,6,7,8,9$, 10, 11] are shown in Fig. 2. The AMS results significantly improve the precision and extend the measurements to the uncharted high energy region.

To examine the energy dependence of the electron flux in a model independent way, the flux spectral index $\gamma$ is calculated from

$$
\gamma=d[\log (\Phi)] / d[\log (E)]
$$

over non-overlapping energy intervals which are chosen to have sufficient sensitivity to the spectral index. The results are presented in Fig. 3 together with the positron results [2]. As seen, both the electron and positron indices decrease (soften) rapidly with energy below $\sim 10 \mathrm{GeV}$, and then they both start increasing (harden) at $>20 \mathrm{GeV}$. In particular, the electron spectral index increases from $\gamma=-3.295 \pm 0.026$ in the energy range $[17.98-27.25] \mathrm{GeV}$ to an average $\gamma=-3.180 \pm 0.008 \mathrm{in}$ the range $[55.58-1400] \mathrm{GeV}$, where it is nearly energy independent. The behavior of the electron and positron spectral indices is distinctly different. 


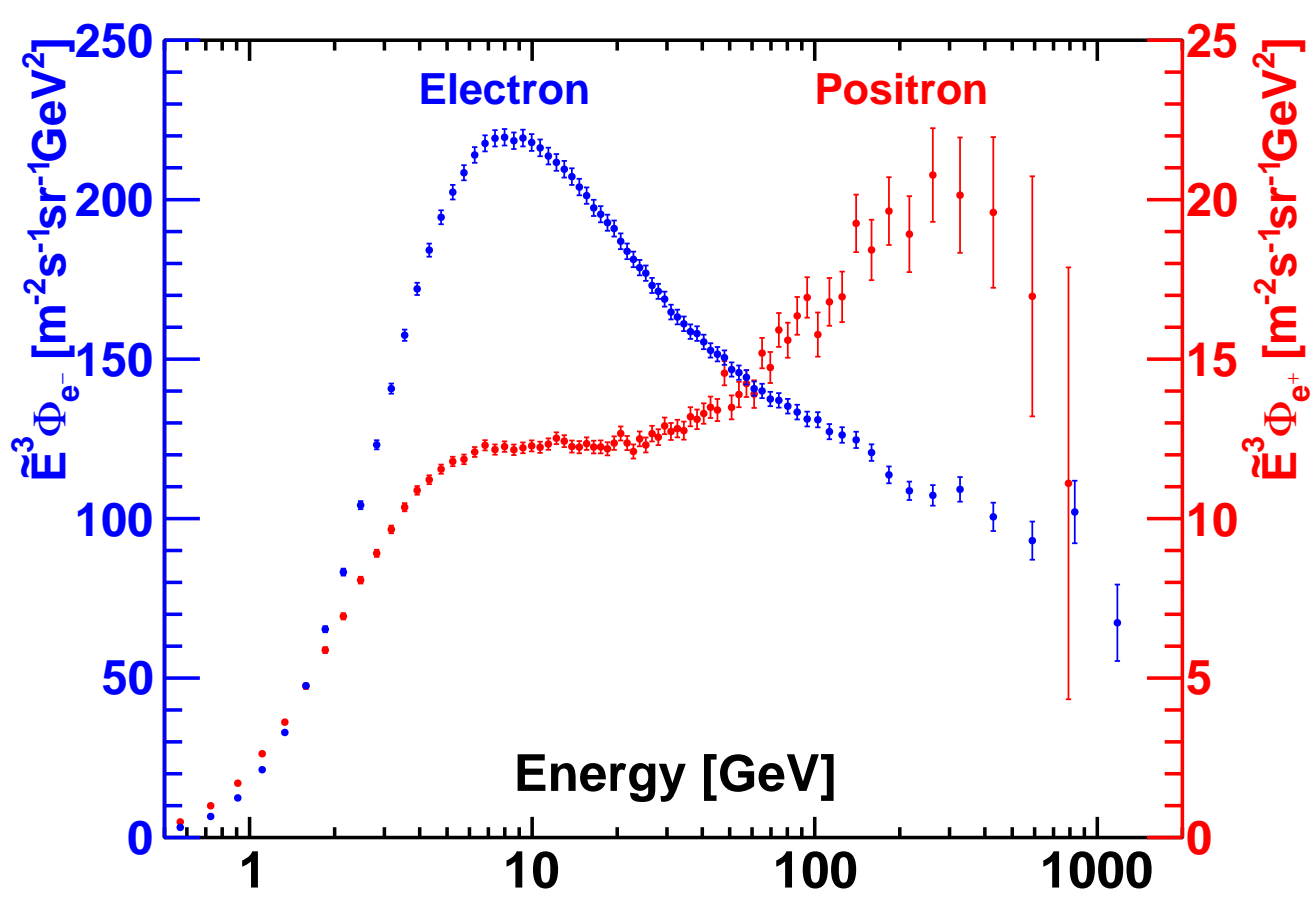

Figure 1: The electron (blue, left axis) and positron (red, right axis) spectra $\left(\tilde{E}^{3} \cdot \Phi_{e^{ \pm}}\right)$. For display purposes the positron data point at $\sim 830 \mathrm{GeV}$ is slightly shifted horizontally to avoid overlap with the electron point. As seen, the electron spectrum has distinctly different magnitude and energy dependence compared to that of positrons

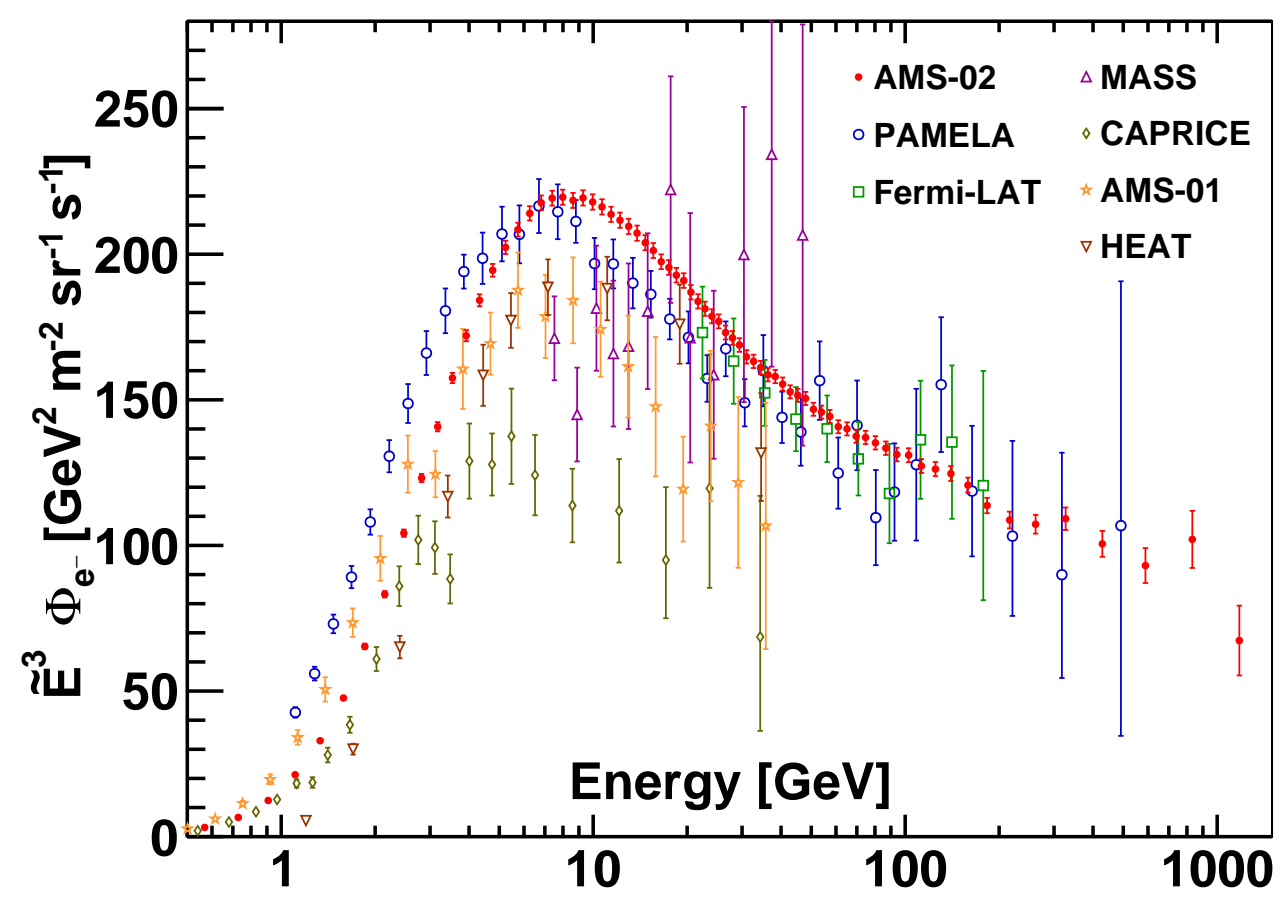

Figure 2: The AMS electron spectrum $\left(\tilde{E}^{3} \cdot \Phi_{e^{-}}\right)$together with earlier experiments. 


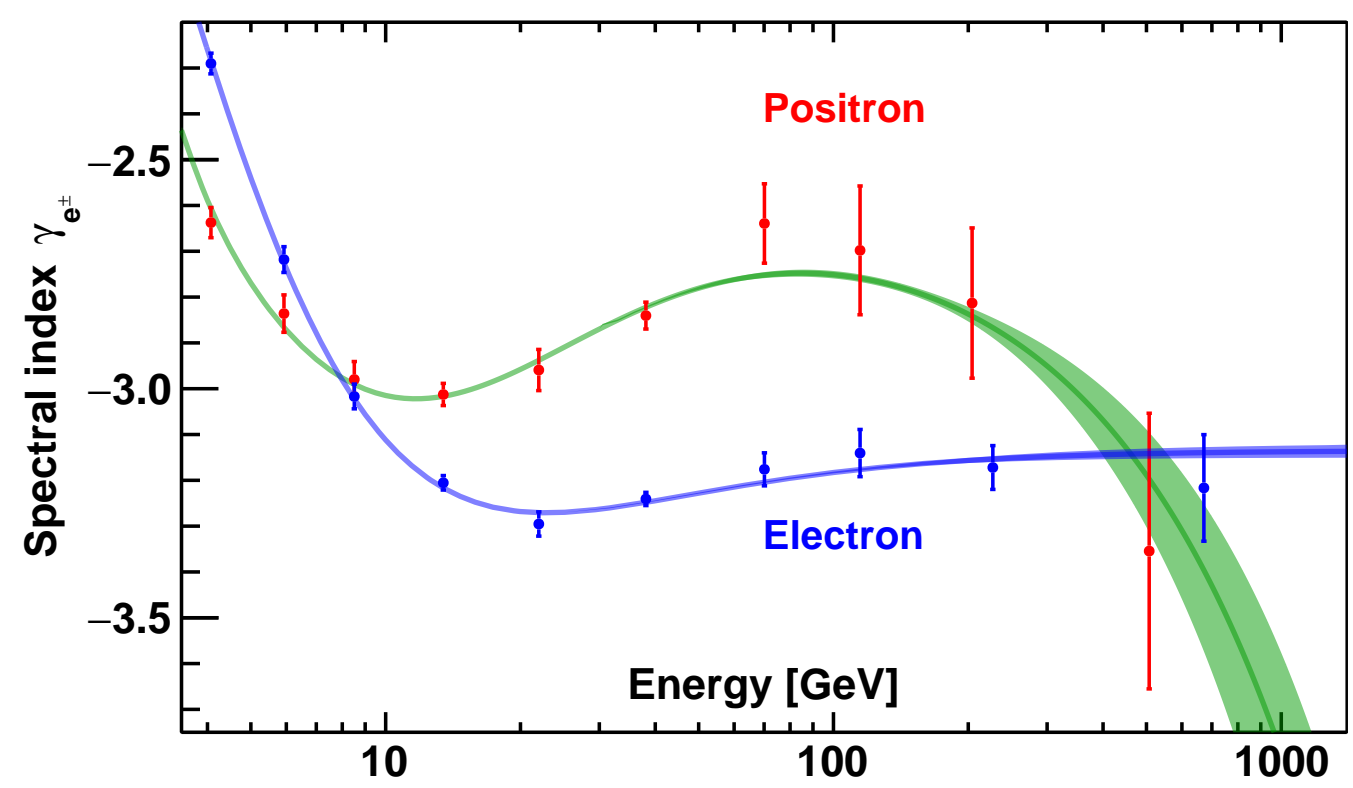

Figure 3: The spectral indices of the electron flux (blue) and positron flux (red) in non-overlapping intervals. They show distinctly different behavior. The blue band represents the 68\% C.L. interval of the fit of Eq. (3.5) to the electron flux (see text). The green band represents the $68 \%$ C.L. interval of a fit to the positron flux [Eq. (4) in Ref. [2]].

To determine the transition energy $E_{0}$ where the change of the electron spectral index occurs, we use a double power law approximation

$$
\Phi_{e^{-}}(E)= \begin{cases}C(E / 20.04 \mathrm{GeV})^{\gamma}, & E \leq E_{0} \\ C(E / 20.04 \mathrm{GeV})^{\gamma}\left(E / E_{0}\right)^{\Delta \gamma}, & E>E_{0}\end{cases}
$$

A fit to data is performed in the energy range $[20.04-1400] \mathrm{GeV}$. The energy range boundary of $20.04 \mathrm{GeV}$ is chosen to be above the observed range of time dependence based on the analysis of Ref. [2]. The results are presented in Fig. 4. The fit yields $E_{0}=42.1_{-5.2}^{+5.4} \mathrm{GeV}$ and $\Delta \gamma=0.094 \pm$ 0.014 with $\chi^{2} /$ d.o.f. $=17.9 / 36$. The energy $E_{0}$ corresponds to the beginning of spectral hardening compared to the lower energy trends. The spectral index change by $\Delta \gamma$ is clearly visible in Fig. 4. Note that the choice of the value $20.04 \mathrm{GeV}$ does not affect the fitted values of $E_{0}, \gamma$, and $\Delta \gamma$.

To investigate the existence of a finite energy cutoff as seen in the positron flux [2], the electron flux is fitted starting from the lower boundary of the $E_{0}$ bin $[41.61-44.00] \mathrm{GeV}$ with

$$
\Phi_{e^{-}}(E)=C_{s}(E / 41.61 \mathrm{GeV})^{\gamma_{s}} \exp \left(-E / E_{s}\right)
$$

A fit to data in the energy range $[41.61-1400] \mathrm{GeV}$ yields the inverse cutoff energy $1 / E_{S}=$ $0.00_{-0.00}^{+0.08} \mathrm{TeV}^{-1}$ with $\chi^{2} /$ d.o.f. $=15.2 / 23$. A study of the cutoff significance shows that $E_{s}<$ $1.9 \mathrm{TeV}$ is excluded at the $5 \sigma$ level. These results are presented in Fig. 5.

New sources of high energy positrons, such as dark matter, may also produce an equal amount of high energy electrons. To test the consistency of this assumption with the data in the energy range $[41.61-1400] \mathrm{GeV}$, the electron flux is parametrized as a sum of a power law component 


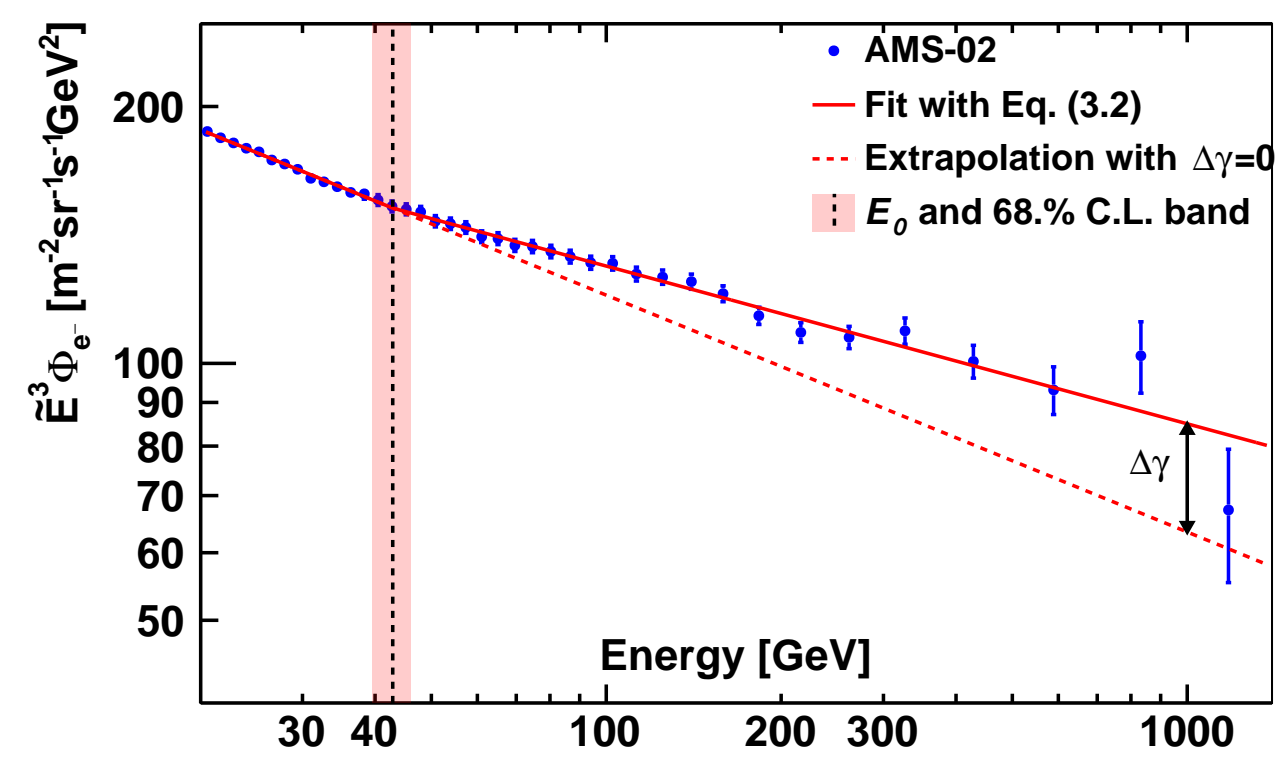

Figure 4: A double power law fit of Eq. (3.2) to the electron flux in the energy range [20.04 - 1400] GeV. The blue data points are the measured electron flux values scaled by $\tilde{E}^{3}$. The fitted function is represented by the solid red line. The vertical dashed line and the red band correspond to the value and the error of the energy $E_{0}$ where the change of the spectral index occurs and $\Delta \gamma=0.094 \pm 0.014$ is the magnitude of the spectral index change, see Eq. (3.2). The dashed red line is the extrapolation of the power law below $E_{0}$ into the higher energy region (or $\Delta \gamma=0$ in Eq. (3.2)).

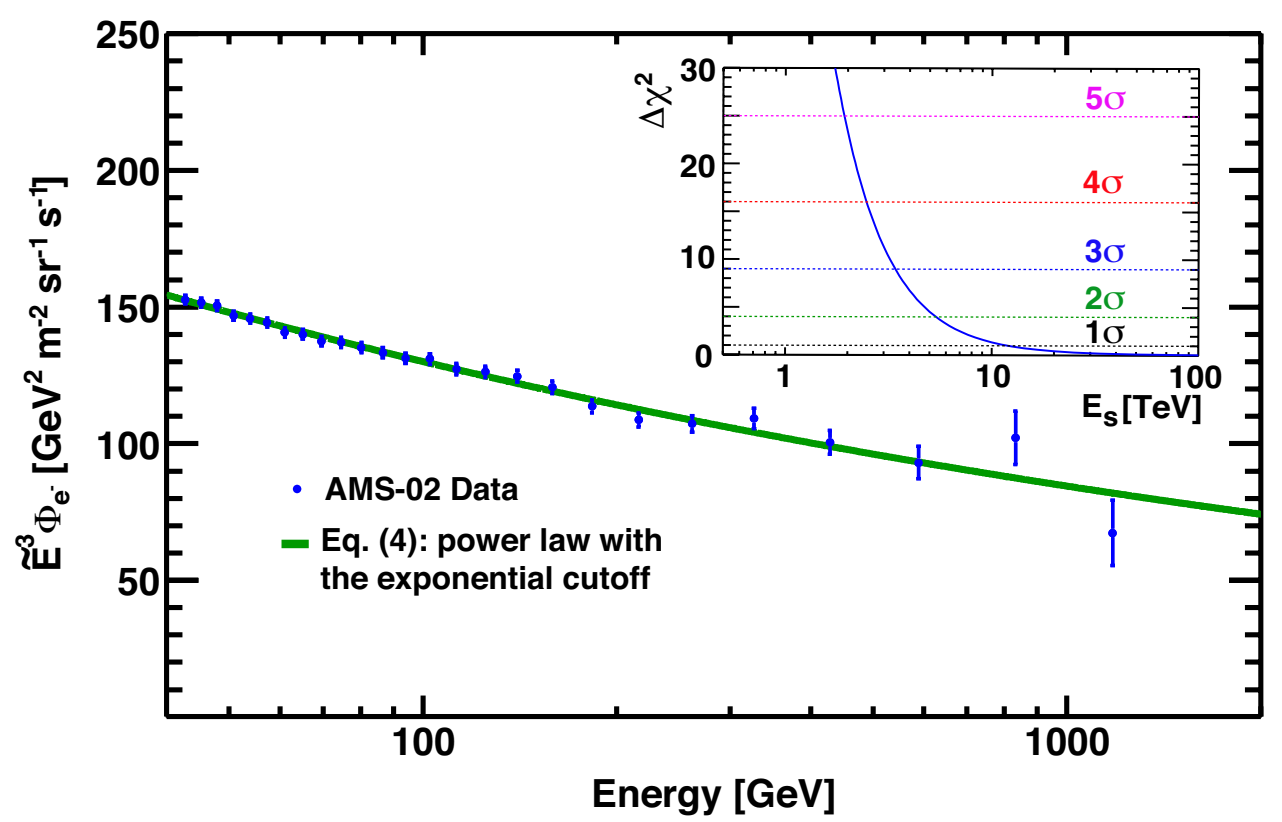

Figure 5: The fit of Eq. 3.3 to the electron flux data in the energy range $[41.61-1400] \mathrm{GeV}$. The insert shows the study of the significance of the $1 / E_{s}$ measurement by varying all three fit parameters in Eq. 3.3 to find the minimal $\Delta \chi^{2}$ corresponding to $E_{s}$ values from 1 to $100 \mathrm{TeV}$. The blue curve shows the dependence of $\Delta \chi^{2}$ on $E_{s}$ and the horizontal dashed lines show different significance levels from 1 to $5 \sigma$. As seen, $E_{s}$ values below $1.9 \mathrm{TeV}$ are excluded at the $5 \sigma$ level. 
and the identical source term as for positrons [2]:

$$
\Phi_{e^{-}}(E)=C_{e^{-}}\left(E / E_{1}\right)^{\gamma_{e^{-}}}+f_{e^{-}} C_{s}^{e^{+}}\left(E / E_{2}\right)^{\gamma_{s}^{+}} \exp \left(-E / E_{s}^{e^{+}}\right) .
$$

The values of the source term parameters $C_{s}^{e^{+}}, \gamma_{s}^{e^{+}}, E_{2}$, and $E_{s}^{e^{+}}$are taken from Ref. [2]. The fit yields $\chi^{2} /$ d.o.f. $=15.5 / 24$ if $f_{e^{-}}$is fixed to 1 , and $\chi^{2} /$ d.o.f. $=15.2 / 24$ if $f_{e^{-}}$is fixed to 0 (i.e. no contribution from positron source term). The analysis shows that AMS electron data are consistent both with the charge symmetric positron source term [2] $\left(f_{e^{-}}=1\right.$ in Eq. (3.4)) and also with the absence of such a term $\left(f_{e^{-}}=0\right)$.

Most surprisingly, we found that in the entire energy range $[0.5-1400] \mathrm{GeV}$ the electron flux is well described by the sum of two power law components:

$$
\Phi_{e^{-}}(E)=\frac{E^{2}}{\hat{E}^{2}}\left[1+\left(\hat{E} / E_{t}\right)^{\Delta \gamma_{t}}\right]^{-1}\left[C_{a}\left(\hat{E} / E_{a}\right)^{\gamma_{a}}+C_{b}\left(\hat{E} / E_{b}\right)^{\gamma_{b}}\right] .
$$

The low energy transition term, $\left[1+\left(\hat{E} / E_{t}\right)^{\Delta \gamma_{t}}\right]^{-1}$, is introduced to account for the complex spectral behavior of the electron flux at energies below $\sim 10 \mathrm{GeV}[12,13]$. The two components, $a$ and $b$, correspond to two power law functions with corresponding normalization factors $C_{a}$ and $C_{b}$, and spectral indices $\gamma_{a}$ and $\gamma_{b}$. To account for solar modulation effects, the force-field approximation [14] is used, with the energy of particles in the interstellar space $\hat{E}=E+\varphi_{e^{-}}$and the effective modulation potential $\varphi_{e^{-}}$. The result of the fit is presented in Fig. 6 . The energy dependence of the electron spectral index corresponding to the results of the fit of Eq. (3.5) is shown in Fig. 3 as a blue $68 \%$ C.L. band.

In the energy range $[0.5-1400] \mathrm{GeV}$ the sum of two power law functions with the transition term provides an excellent description of the data. These functions are very different in shape and in magnitude from those describing the positron flux [2]. The power law $a$ contribution exceeds the expected secondary electron or positron production by a factor of $\sim 20$ (see Ref. [15]). The power law $b$ contribution, which dominates the electron flux at high energies $>40 \mathrm{GeV}$, significantly exceeds the magnitude of the positron source term [2].

The observations discussed above are clear evidences that most cosmic-ray electrons originate from different sources than cosmic-ray positrons.

\section{Conclusion}

We have presented the high statistics precision measurements of the electron flux from $0.5 \mathrm{GeV}$ to $1.4 \mathrm{TeV}$, with detailed study of systematic uncertainties based on a data sample of 28.1 million electrons. In the entire energy range the electron and positron spectra have distinctly different magnitudes and energy dependences. The electron flux exhibits a significant excess starting from $42.1_{-5.2}^{+5.4} \mathrm{GeV}$ compared to the lower energy trends, but the nature of this excess is different from the positron flux excess above $25.2 \pm 1.8 \mathrm{GeV}$. Contrary to the positron flux, which has an exponential energy cutoff of $810_{-180}^{+310} \mathrm{GeV}$, at the $5 \sigma$ level the electron flux does not have an energy cutoff below $1.9 \mathrm{TeV}$. In the entire energy range from $0.5 \mathrm{GeV}$ to $1.4 \mathrm{TeV}$ the electron flux is well described by the sum of two power law components. The different behavior of the cosmic-ray electrons and positrons measured by AMS is clear evidence that most high energy electrons originate from different sources than high energy positrons. 


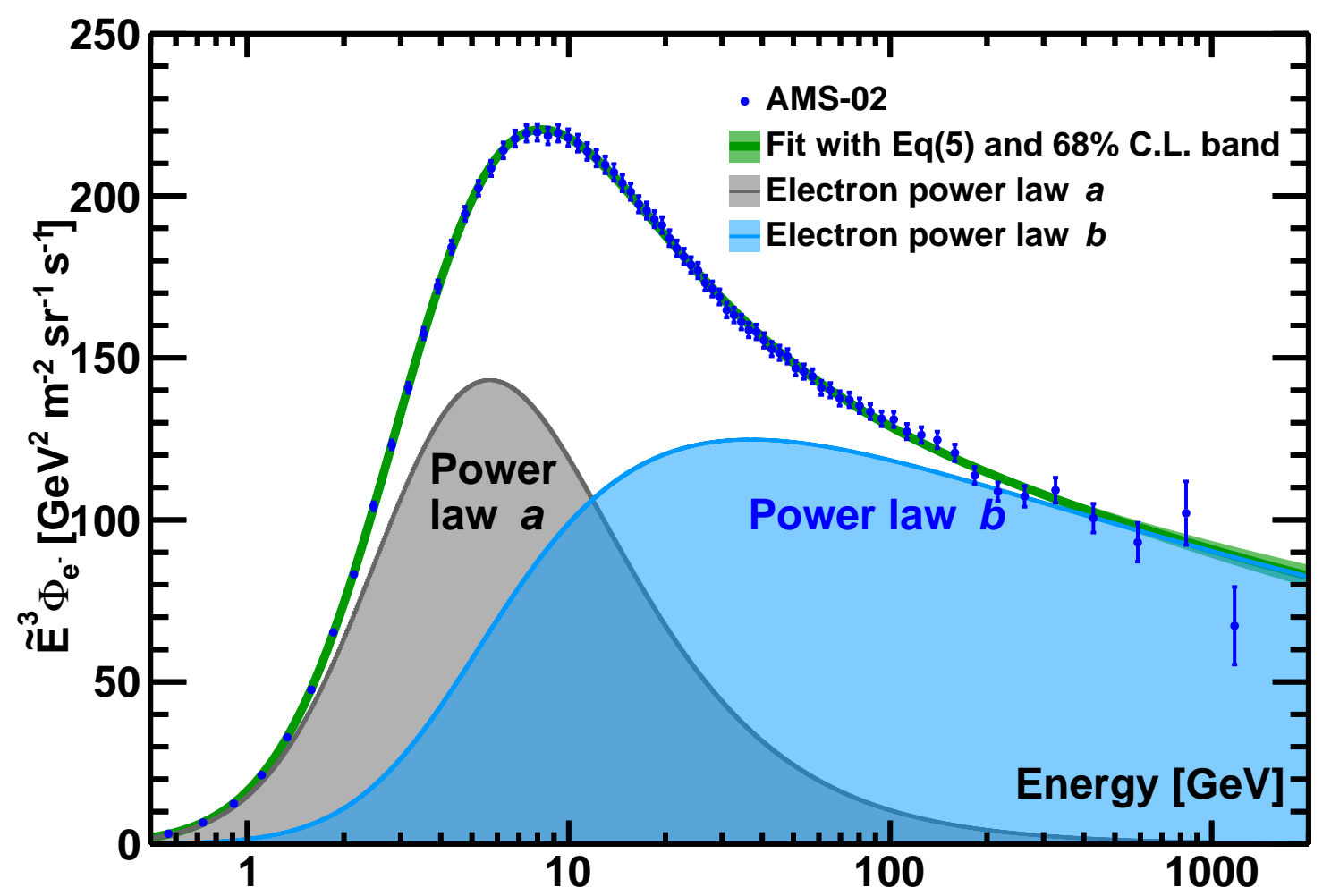

Figure 6: The two power law fit of Eq. (3.5) to the electron flux data in the energy range $[0.5-1400] \mathrm{GeV}$ with the $68 \%$ C.L. (green band). The two power law components $a$ and $b$ of Eq. (3.5) are represented by the gray and blue areas, respectively.

\section{Acknowledgments}

This work has been supported by acknowledged person and institutions in Ref. [2].

\section{References}

[1] M. Aguilar et al., Phys. Rev. Lett. 110, 141102 (2013);

[2] M. Aguilar et al., Phys. Rev. Lett. 122, 041102 (2019);

[3] M. Aguilar et al., Phys. Rev. Lett. 122, 101101 (2019);

[4] G.D. Lafferty and T.R. Wyatt, NIM A 355, 541 (1995). We have used Eq. (6) with $\tilde{E} \equiv x_{l w}$.

[5] J. Alcaraz et al., Phys. Lett. B 484, 10 (2000).

[6] O. Adriani et al., Phys. Rev. Lett. 106, 201101 (2011).

[7] M. Ackermann et al., Phys. Rev. Lett. 108, 011103 (2012).

[8] C. Grimani et al., Astron. Astrohys. 392, 287 (2002).

[9] M. Boezio et al., Adv. Space Res. 27, 669 (2001).

[10] M. Aguilar et al., Phys. Lett. B 646, 145 (2007). 
[11] S.W. Barwick et al., Astrophys. J. 498, 779 (1998); M.A. DuVernois et al., Astrophys. J. 559, 296 (2001); J.J. Beatty et al., Phys. Rev. Lett. 93, 241102 (2004).

[12] M. Aguilar et al., Phys. Rev. Lett. 121, 051102 (2018).

[13] Ł. Stawarz, V. Petrosian, and R. D. Blandford, Astrophys. J. 710, 236 (2010).

[14] R. A. Caballero-Lopez and H. Moraal, J. Geophys. Res. 109, A01101 (2004); L. Gleeson and W. Axford, Astrophys. J. 154, 1011 (1968).

[15] I. V. Moskalenko and A. W. Strong, Astrophys. J. 493, 694 (1998); A. E. Vladimirov, S. W. Digel, G. Jóhannesson, P. F. Michelson, I. V. Moskalenko, P. L. Nolan, E. Orlando, T. A. Porter, and A. W. Strong, Comput. Phys. Commun. 182, 1156 (2011). 